\title{
Predictors of Short- and Long-Term Mortality in Ischemic Stroke: A Community-Based Study in Brno, Czech Republic
}

\author{
Tomas Bryndziara,b, c Dominika Matyskovaa, b Petra Sedovaa, b, c, d, e \\ Silvie Belaskova ${ }^{a}, f$ Miroslav Zvolskyg Josef Bednarik ${ }^{b}, h \quad$ Robert D. Brown ${ }^{d}$ \\ Robert Mikulik ${ }^{a, b, c}$
}

\begin{abstract}
a International Clinical Research Center, St. Anne's University Hospital, Brno, Czech Republic; b Faculty of Medicine, Masaryk University, Brno, Czech Republic; 'Department of Neurology, St. Anne's University Hospital, Brno, Czech Republic; ${ }^{d}$ Department of Neurology, Mayo Clinic, Rochester, MN, USA; ${ }^{\circ}$ Department of Internal Medicine, Hematology and Oncology, University Hospital Brno, Brno, Czech Republic; ${ }^{f}$ Institute of Mathematics and Statistics, Masaryk University, Brno, Czech Republic; IInstitute for Health Information and Statistics of the Czech Republic,

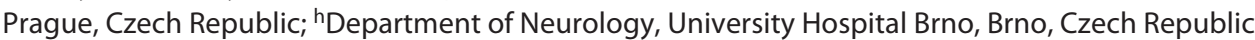

\section{Keywords}

Czech Republic $\cdot$ Ischemic stroke $\cdot$ Mortality

\begin{abstract}
Background and Objective: Short- and long-term mortality following ischemic stroke (IS) and their predictors have not been defined in the Czech population, and studies on longterm mortality are largely missing for the populations of Central Europe. Methods: Using the National Register of Hospitalized Patients and the Czech National Mortality Registry, we analyzed data on 1-month, 1-year, and 3-year allcause mortality for patients admitted with IS to any of the 4 hospitals with a certified stroke unit in Brno, Czech Republic, in 2011. We reviewed discharge summaries and recorded potential factors impacting mortality after the index stroke event. Using univariate and multivariable analyses, we identified predictors of mortality at all 3 time points. Results: In our multivariable model, statin use (odds ratio [OR] 0.095, $p$ $<0.0001$ ), age at stroke (OR 1.03, $p=0.0445)$, and admission
\end{abstract}

karger@karger.com www.karger.com/ced

Karger $\frac{1}{\%}$
(C) 2021 The Author(s)

Published by S. Karger AG, Basel

This is an Open Access article licensed under the Creative Common Attribution-NonCommercial-4.0 International License (CC BY-NC) (http://www.karger.com/Services/OpenAccessLicense), applicable to the online version of the article only. Usage and distribution for commercial purposes requires written permission.
National Institutes of Health Stroke Scale (NIHSS) score (OR 1.16, $p<0.0001)$ predicted 1-month mortality, while statin use (OR 0.43, $p=0.0004$ ), history of cardiac failure (OR 2.17, $p=0.0137$ ), age at stroke (OR 1.07, $p<0.0001)$, and admission NIHSS score (OR 1.14, $p<0.0001$ ) predicted 1-year mortality. Statin use (OR $0.54, p=0.0051$ ), history of cardiac failure (OR $2.13, p=0.0206$ ), age at stroke (OR 1.07, $p<0.0001$ ), and admission NIHSS score (OR 1.11, $p<0.0001$ ) also predicted 3-year mortality. Conclusions: Our study is the first to report data on short- and long-term mortality rates and their predictors in patients hospitalized with IS in the Czech population. Our results indicate that mortality rates and predictors of mortality are consistent with those reported in studies from other populations throughout the world.

(c) 2021 The Author(s).

Published by S. Karger AG, Basel

Tomas Bryndziar and Dominika Matyskova contributed equally to this work. 


\section{Introduction}

Despite advances in prevention and treatment, ischemic stroke (IS) remains a significant cause of disability and death worldwide [1]. While short-term mortality and case fatality rates of IS have been described previously, such data are scarce for populations in Central Europe [2-6]. One-month case fatality rates for IS have been reported to be between $5 \%$ and $37.6 \%$ worldwide and $14.2 \%$ in Europe [2]. Data on long-term prognosis of IS and its determinants are available from long-running epidemiological studies in the USA $[7,8]$ and Western Europe [9, 10], but similar long-term data from Central Europe are not widely available $[5,11]$.

This study is part of our ongoing efforts to better characterize the epidemiology of stroke in the Czech Republic $[12,13]$ in a setting where the estimated burden of stroke is high [1], but resources for large-scale epidemiological projects have been limited. We assess 1-month, 1-year, and 3-year all-cause mortality rates in patients hospitalized with IS in a community-based study in Brno, Czech Republic, in 2011, and identify risk factors predicting short- and long-term mortality after stroke.

\section{Methods}

This study uses data from two Czech national administrative databases - the National Register of Hospitalized Patients (NRHOSP) and the Czech National Mortality Registry (CNMR).

We have described both databases previously $[12,14]$. In short, the NRHOSP prospectively collects data about all hospital admissions in the Czech Republic. It contains diagnostic codes (using the International Classification of Diseases, Tenth Revision [ICD-10]) for one main and up to four secondary discharge diagnoses that are retrieved from patients' discharge summaries. We have previously validated the accuracy of the diagnostic codes for different stroke types in this database and found the accuracy of coding for IS (ICD-10 code I63 [cerebral infarction]) to be $82 \%$ (95\% CI: 74$89 \%$ ) [12]. The CNMR collects information regarding all deaths in the Czech Republic [14].

For this study, we searched the NRHOSP for all patients with discharge diagnosis of I63 admitted to any of the 4 hospitals with a certified stroke unit in Brno, Czech Republic (Hospital of the Merciful Brothers, Military Hospital Brno, St. Anne's University Hospital, and the University Hospital Brno) between January 1, 2011, and December 31, 2011. The year 2011 was selected due to the availability of accurate demographic data from the 2011 Czech Population and Housing Census. For each patient identified, we requested his or her complete discharge summary from the admitting hospital. Two neurologists independently reviewed the discharge summaries, verified the discharge diagnosis of IS, and determined the IS subtype using TOAST criteria [15]. Stroke was defined using the standard WHO definition as "rapidly developed clinical signs of focal (or global) disturbance of cerebral function, lasting $>24$ h or leading to death, with no apparent cause other than of vascular origin" [16]. In case of a disagreement, a senior neurologist reviewed the case and made the final decision. Only patients with permanent address in Brno, Czech Republic (as listed in their discharge summary), were included in this study. Table 1 summarizes the case selection criteria.

To identify the patients who died, regardless of cause, following a diagnosis of IS and to calculate all-cause mortality rates, we linked the data from the hospitalization registry (NRHOSP) with the data from the mortality registry (CNMR). We used the same approach as in our previous study of case fatality rates of hemorrhagic stroke [14]. Using the date of admission in the NRHOSP and the date of death in the CNMR, we calculated 1-month, 1-year, and 3-year all-cause mortality rates.

To assess potential predictors of mortality, we reviewed all discharge summaries and recorded data including history of cerebral infarction, intracerebral hemorrhage, heart failure, myocardial infarction, atrial fibrillation, hypertension, diabetes mellitus, hyperlipidemia, dementia, medication use, and the National Institutes of Health Stroke Scale (NIHSS) score at admission. Using clinical, laboratory, and imaging data from the discharge summaries, 2 stroke neurologists independently determined the IS subtype using TOAST criteria (large-artery atherosclerosis, cardioembolism, small-artery occlusion, acute stroke of other determined etiology, and stroke of undetermined etiology) [15]. In case of a disagreement, a senior stroke neurologist made the final determination.

Data are represented in an appropriate way with respect to their distribution. A univariate analysis was performed to assess for potential risk factors of IS mortality. For univariate analysis, Fisher's exact test, two-sample $t$-test, and Wilcoxon rank-sum test have been used to detect potential statistically significant risk factors. Risk factors have been selected based on statistical significance and clinical relevance. The selected risk factors, together with additional clinically relevant factors, were then used in the multivariable models. Proc logistic and stepwise selection was used to input factors into the model. The significance level for imputation was 0.3 for forward selection and 0.35 for backward selection. All results were rated for 3 time periods - 1 month, 1 year, and 3 years. Hypotheses were tested on a significant level alpha $=0.05$ and statistical analyses were performed by SAS statistical software (Copyright@ 2017, SAS Institute Inc., Cary, NC, USA).

\section{Results}

A total of 618 patients with IS were included. Mean age at stroke onset was 74 years $(\mathrm{SD}=12)$ and $47.7 \%$ were women. Median admission NIHSS was $4(\mathrm{IQR}=7)$. Of the 618 patients, 75 died within 1 month, 182 within 1 year, and 268 within 3 years following the index stroke, yielding mortality rates of, respectively, $12.1 \%, 29.4 \%$, and $43.3 \%$.

In the univariate analysis, an increased risk of 1-month and 1-year mortality was predicted by history of heart failure, myocardial infarction, and atrial fibrillation, and by cardioembolic IS subtypes; history of hyperlipidema 
Table 1. Case selection criteria for inclusion in this study
Discharge diagnosis of cerebral infarction (163) in the NRHOSP

Stroke events occurring between January 1, 2011, and December 31, 2011

Admission to one of the 4 hospitals with a certified stroke unit in Brno, Czech Republic

Permanent address in Brno, Czech Republic, as listed in the discharge summary

Two study neurologists independently verified the discharge diagnosis of cerebral infarction (163) by a detailed review of hospital discharge records. NRHOSP, national register of hospitalized patients. and small vessel disease IS subtype were associated with a reduced mortality. An increase in 3-year mortality was predicted by a history of cerebral infarction, heart failure, myocardial infarction, atrial fibrillation, diabetes mellitus, and dementia, and cardioembolic IS subtype; a history of hyperlipidemia and large vessel atherothrombosis, small vessel disease, and "other" IS subtypes were associated with a reduced mortality at 3 years. Detailed results of the univariate analysis are given in Table 2.

In the multivariable model, age at stroke, NIHSS at hospital admission, and statin use (reduction in mortality) predicted 1-month mortality, while history of cardiac failure, age at stroke, NIHSS at hospital admission and statin use (reduction in mortality) predicted both 1-year and 3-year mortality. Detailed results of the multivariate analysis are given in Table 3.

\section{Discussion}

The IS mortality rates reported herein for Brno, Czech Republic are consistent with the short- and long-term mortality rates reported from western countries and other sites in Europe. The 1-month mortality rate of $12.1 \%$ is similar to the $14.2 \%$ estimate of 1 -month case fatality rate overall in Europe, reported by a meta-analysis of 43 population-based studies from different European populations [2]. Our results also compare similarly with the 1-month and 1-year case fatality rates reported from other countries in Central Europe. In Poland, 1-month case fatality rates have been reported by a community- and registry-based study to be 38\% between 1991 and 1992 [4], decreasing to $19.5 \%$ and $15.9 \%$ between 2006 and $2015(17.7 \%$ in 2011$)$ in a regional registry-based study [6], and decreasing from $17.09 \%$ to $16.36 \%$ between 2009 and 2013 (16.63\% in 2011) in a nationwide registry-based study [5]. In Hungary, a study based on a nationwide stroke registry found 9.2\% 28-day case fatality rate in 1998 [3], and another nationwide registry-based study (part of a multinational EuroHOPE epidemiological study) reported 30 -day case fatality to be $16.3 \%$ in 2007 [11]. The 1-year mortality rate reported in the current study is very similar to the $32.52 \% 12$-month follow-up mortality [5] and the $31.9 \% 12$-month case fatality [6] reported for 2011 in 2 recent studies from Poland, as well as the $31.0 \% 365$-day all-cause case fatality reported in a study from Hungary [11]. While we did not find any studies reporting longer-term mortality for countries of Central Europe, our 3-year mortality rate, $43.3 \%$, is consistent with older long-term follow-up data reported from $\mathrm{Ox}$ fordshire, UK (52\% actuarial risk of death at 5 years after IS) [9] and Rochester, MN, USA (46\% survival at 5 years after IS) [8].

History of hyperlipidemia was associated with lower mortality at all 3 time points in our univariate analysis. This is consistent with the results of a recent study based on the Greater Cincinnati/Northern Kentucky Stroke Study, which has shown that hyperlipidemia in IS patients was associated with reduced 30-day, 1-year, and 3-year mortality, regardless of statin use (but with added long-term survival benefit in patients using them) [17]. While the effect of hyperlipidemia disappeared in our multivariable model, this model showed a positive effect of statin use on decreasing mortality at all 3 time points. These results are also consistent with those observed in multiple previous studies, where statin use was associated with lower long-term post-stroke mortality [17-19]. Mechanisms where statins may affect survival following IS include improving cerebral perfusion and collateral blood supply, direct neuroprotection, stabilization of atherosclerotic plaques, and improved angio-, neuro-, and synaptogenesis [20]. Alternatively, history of statin use may be an indicator of health-seeking behavior in individuals with such history, which might account for the better outcomes detected in this study. However, the retrospective design and relatively limited number of subjects precluded us from establishing which explanation is more likely for the observed effects.

Cardiovascular risk factors were significant predictors of both short- and long-term mortality in both our uni- 
Table 2. Results of the univariate analysis

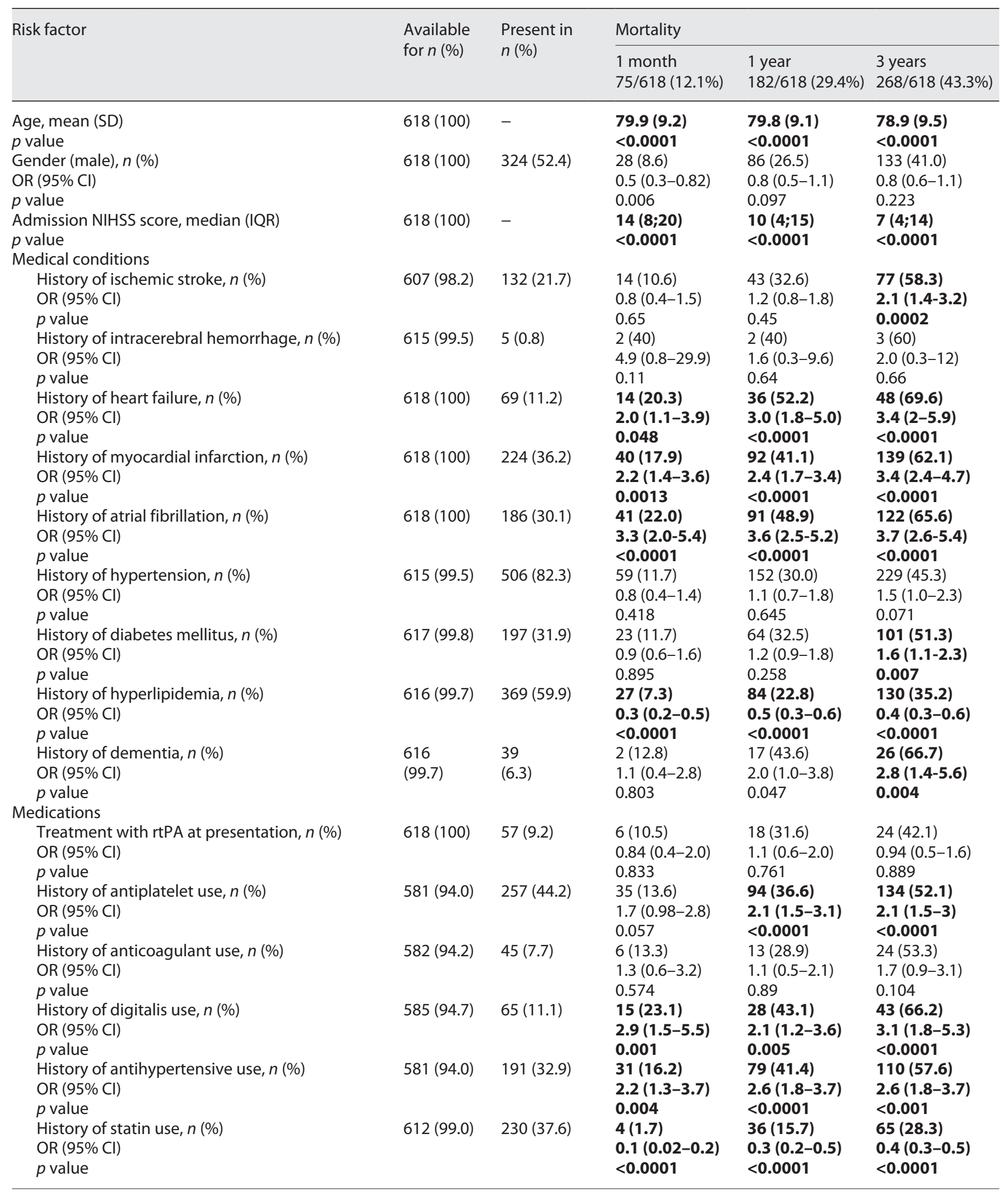

Predictors of Short- and Long-Term Ischemic Stroke Mortality 
Table 2 (continued)

\begin{tabular}{|c|c|c|c|c|c|}
\hline \multirow[t]{2}{*}{ Risk factor } & \multirow{2}{*}{$\begin{array}{l}\text { Available } \\
\text { for } n(\%)\end{array}$} & \multirow{2}{*}{$\begin{array}{l}\text { Present in } \\
n(\%)\end{array}$} & \multicolumn{3}{|l|}{ Mortality } \\
\hline & & & $\begin{array}{l}1 \text { month } \\
75 / 618(12.1 \%)\end{array}$ & $\begin{array}{l}1 \text { year } \\
182 / 618(29.4 \%)\end{array}$ & $\begin{array}{l}3 \text { years } \\
268 / 618(43.3 \%)\end{array}$ \\
\hline \multicolumn{6}{|l|}{ TOAST subtype } \\
\hline TOAST subtype: large-artery atherosclerosis, $n(\%)$ & $618(100)$ & $172(27.8)$ & $17(9.9)$ & $41(23.8)$ & $62(36.1)$ \\
\hline OR $(95 \% \mathrm{Cl})$ & & & $0.7(0.4-1.3)$ & $0.7(0.5-1.0)$ & $0.7(0.5-0.9)$ \\
\hline$p$ value & & & 0.337 & 0.062 & 0.030 \\
\hline TOAST subtype: cardioembolism, $n$ (\%) & $618(100)$ & $214(34.6)$ & $45(21.0)$ & $101(47.2)$ & $134(62.6)$ \\
\hline OR $(95 \% \mathrm{Cl})$ & & & $3.3(2.0-5.5)$ & $3.6(2.5-5.1)$ & $3.4(2.4-4.8)$ \\
\hline$p$ value & & & $<0.0001$ & $<0.0001$ & $<0.0001$ \\
\hline TOAST subtype: small-artery occlusion, $n$ (\%) & $618(100)$ & $146(23.6)$ & $3(2.1)$ & $18(12.3)$ & $36(24.7)$ \\
\hline $\mathrm{OR}(95 \% \mathrm{Cl})$ & & & $0.1(0.0-0.4)$ & $0.3(0.2-0.4)$ & $0.3(0.2-0.5)$ \\
\hline$p$ value & & & $<0.0001$ & $<0.0001$ & $<0.0001$ \\
\hline TOAST subtype: other determined etiology, $n(\%)$ & $618(100)$ & $44(7.1)$ & $2(4.6)$ & $7(15.9)$ & $12(27.3)$ \\
\hline OR $(95 \% \mathrm{Cl})$ & & & $0.3(0.1-1.4)$ & $0.4(0.2-1.0)$ & $0.5(0.2-0.9)$ \\
\hline$p$ value & & & 0.148 & 0.041 & 0.027 \\
\hline TOAST subtype: undetermined etiology, $n(\%)$ & $618(100)$ & $43(7.0)$ & $8(18.6)$ & $15(34.9)$ & $24(55.8)$ \\
\hline OR $(95 \% \mathrm{Cl})$ & & & $1.7(0.8-3.9)$ & $1.3(0.7-2.5)$ & $1.7(0.9-3.2)$ \\
\hline$p$ value & & & 0.220 & 0.488 & 0.110 \\
\hline
\end{tabular}

Statistically significant results are in bold. Mortality rates were calculated as proportions of patients who died within 1 month, 1 year, and 3 years. For each risk factor (column 1) and each period (columns 4, 5, and 6), we compared the patients who died with those that survived. The $p$ values were calculated using Fisher's exact test for comparison of proportions (categorical variables), two-sample $t$-test for comparison of means (age), and Wilcoxon rank-sum test for comparison of medians (admission NIHSS). Cl, confidence interval; IQR, interquartile range; NIHSS, national institutes of health stroke scale; OR, odds ratio; rtPA, recombinant tissue plasminogen activator; SD, standard deviation.

variate and multivariable analyses. Atrial fibrillation was associated with increased mortality in our univariate analysis, with the odds of history of atrial fibrillation over 3 times higher in patients who died within all 3 time points. This is consistent with the results of previous studies, which found that atrial fibrillation increased mortality after IS in hospital [21-23], in the short term [24-26], in the medium term [26], as well as in the long term [8, $24,25,27]$. History of heart failure was also associated with higher mortality rates in both univariate and multivariable models. This is consistent with observations from other studies $[7,8]$.

Our study has several limitations. First, the retrospective design of our study and its reliance on information obtained from discharge summaries limited our ability to gather comprehensive data for all potential predictors of mortality, thus limiting our analyses to variables available in a sufficient number of subjects and described in a sufficient level of detail in the discharge summaries. We thus had to analyze as categorical variables factors that could potentially be analyzed as continuous (e.g., hypertension, hyperlipidemia) or ordinal (e.g., severity of heart failure) variables in a prospective study. However, these limitations are inherent to all retrospective, chart-review studies. Second, due to the inclusion of only hospitalized cases of IS, our data are generalizable to hospitalized IS patients only. While we do not expect the number of cases missed for this reason to be high (all patients with a suspected stroke are required to be transported into a hospital with a certified stroke unit as per the guidelines applicable in the whole Czech Republic), we cannot exclude the possibility that the mortality and its predictors in nonhospitalized patients with "silent" or very mild IS differ significantly from those of patients with IS admitted to a hospital. There may have also been a limited number of patients with immediately fatal IS who would not have been hospitalized. Third, because we assessed the patients' outcomes using exclusively data from CNMR, our analysis was limited to the binary outcome of alive versus dead at the studied time points. Given the retrospective design and no in-person follow-up in our study, assessing other outcomes, such as the degree of disability or functional independence, was not feasible. Finally, for patients with large-artery atherosclerosis, we did not analyze the 
Table 3. Results of the multivariable model

\begin{tabular}{|c|c|c|c|}
\hline \multirow[t]{2}{*}{ Risk factor } & \multicolumn{3}{|l|}{ Mortality } \\
\hline & 1 month & 1 year & 3 years \\
\hline \multicolumn{4}{|l|}{ Statin use } \\
\hline OR $(95 \% \mathrm{Cl})$ & $0.095(0.032-0.28)$ & $0.43(0.27-0.68)$ & $0.54(0.35-0.83)$ \\
\hline$p$ value & $<0.0001$ & 0.0004 & 0.0051 \\
\hline \multicolumn{4}{|c|}{ History of heart failure } \\
\hline OR $(95 \% \mathrm{Cl})$ & $x$ & $2.17(1.16-4)$ & $2.13(1.12-4)$ \\
\hline$p$ value & $x$ & 0.0137 & 0.0206 \\
\hline \multicolumn{4}{|c|}{ History of atrial fibrillation } \\
\hline OR $(95 \% \mathrm{Cl})$ & $x$ & $1.54(0.98-2.38)$ & $X$ \\
\hline$p$ value & $x$ & 0.0611 & $x$ \\
\hline \multicolumn{4}{|l|}{ Age at stroke } \\
\hline OR $(95 \% \mathrm{Cl})$ & $1.03(1.001-1.06)$ & 1.07 (1.04-1.09) & $1.07(1.05-1.09)$ \\
\hline$p$ value & 0.0445 & $<0.0001$ & $<0.0001$ \\
\hline \multicolumn{4}{|l|}{ NIHSS } \\
\hline OR $(95 \% \mathrm{Cl})$ & $1.16(1.11-1.20)$ & $1.14(1.10-1.18)$ & $1.11(1.1-1.15)$ \\
\hline$p$ value & $<0.0001$ & $<0.0001$ & $<0.0001$ \\
\hline \multicolumn{4}{|c|}{ History of hyperlipidemia } \\
\hline OR $(95 \% \mathrm{Cl})$ & $x$ & $x$ & $0.75(0.50-1.15)$ \\
\hline$p$ value & $x$ & $x$ & 0.1846 \\
\hline \multicolumn{4}{|l|}{ TOAST subtype } \\
\hline \multicolumn{4}{|l|}{ LAA versus SAO } \\
\hline OR $(95 \% \mathrm{Cl})$ & $0.41(0.11-1.52)$ & $\mathrm{X}$ & $0.79(0.45-1.37)$ \\
\hline$p$ value & 0.1819 & $x$ & 0.3975 \\
\hline \multicolumn{4}{|l|}{ LAA versus CE } \\
\hline OR $(95 \% \mathrm{Cl})$ & $1.25(0.62-2.53)$ & $x$ & $1.60(0.98-2.62)$ \\
\hline$p$ value & 0.5389 & $x$ & 0.0608 \\
\hline
\end{tabular}

Statistically significant results are in bold. CE, cardioembolism; Cl, confidence interval; IS, ischemic stroke; LAA, large-artery atherosclerosis; NIHSS, national institutes of health stroke scale; OR, odds ratio; SAO, small-artery occlusion.

location of the lesion (i.e., extracranial vs. intracranial). This is a limitation because carotid stenosis has been shown to be "a strong and independent predictor of death" [28].

We have been making the effort to improve the quality of stroke care in the Czech Republic, for example, by developing the Registry of Stroke Care Quality (RES-Q) [29]. The current study contributes to this effort with community-level data on rates and predictors of shortand long-term mortality after IS. Prospective studies validating our results on more recent data sets and in different communities in the Czech Republic may be needed to support the translation of our findings into improved national clinical practice guidelines. Additional studies evaluating secondary prevention, adherence to guidelines, and follow-up of high-risk patients in the Czech Republic are currently planned.

\section{Conclusion}

Our study found that the short- and long-term mortality rates and their predictors in patients hospitalized with IS in Brno, Czech Republic, are similar to those previously observed in populations of different geographical regions of the world. It is the first study to report these data for the population of the Czech Republic, and thus provide information that can support better-informed public health policymaking, individual clinical decisionmaking, and prognostication for patients with IS.

\section{Acknowledgments}

We would like to thank Michaela Volna and Jana Baluchova for helping with the data collection. 


\section{Statement of Ethics}

Ethics committees of all participating institutions (Hospital of the Merciful Brothers, Military Hospital Brno, St. Anne's University Hospital, the University Hospital Brno, and the Institute of Health Information and Statistics of the Czech Republic) approved this study (RF. 36v/2014). Informed consent requirement was waived by all the above ethics committees, as this is a retrospective medical record review study.

\section{Conflict of Interest Statement}

Tomas Bryndziar reports employment at Bristol-Myers Squibb outside the submitted work. All other authors have no conflict of interest to declare.

\section{Funding Sources}

Petra Sedova and Robert Mikulik were supported by the COST (European Cooperation in Science and Technology) Association, project no. CA18118, IRENE COST Action - Implementation Re- search Network in Stroke Care Quality and by the project no. LQ1605 from the National Program of Sustainability II and by the IRIS-TEPUS Project No. LTC20051 from the INTER-EXCELLENCE INTER-COST program of the Ministry of Education, Youth and Sports of the Czech Republic.

\section{Author Contributions}

D.M. collected the data. D.M. and T.B. performed literature review. T.B. wrote the manuscript. P.S., R.D.B., and R.M. oversaw the study design and execution. S.B. performed the statistical analysis. M.Z. furnished the national data. All authors revised the manuscript for important intellectual content and approved it for submission.

\section{Data Availability Statement}

The data that support the findings of this study are available upon request from the corresponding author.

\section{References}

1 GBD 2016 Stroke Collaborators. Global, regional, and national burden of stroke, 1990-2016: a systematic analysis for the Global Burden of Disease Study 2016. Lancet Neurol. 2019;18(5):439-58.

2 Zhang R, Wang Y, Fang J, Yu M, Wang Y, Liu G. Worldwide 1 month case fatality of ischaemic stroke and the temporal trend. Stroke Vasc Neurol. 2020;5(4):353-60.

3 Ováry C, Suzuki K, Nagy Z. Regional differences in incidence rates, outcome predictors and survival of stroke. Neuroepidemiology. 2004;23(5):240-6.

4 Weissbein T, Czlonkowska A, Popow J, Ryglewicz D, Hier DB. Analysis of 30 day stroke mortality in a community-based registry in Warsaw, Poland. J Stroke Cerebrovasc Dis. 1994;4(1):63-7.

5 Chwojnicki K, Ryglewicz D, Wojtyniak B, Zagożdżon P, Członkowska A, Jędrzejczyk T, et al. Acute ischemic stroke hospital admissions, treatment, and outcomes in Poland in 2009-2013. Front Neurol. 2018;9:134.

6 Gierlotka M, Labuz-Roszak B, Wojtyniak B, Lasek-Bal A, Zdrojewski T, Adamczyk-Sowa $\mathrm{M}$, et al. Early and one-year outcomes of acute stroke in the industrial region of Poland during the decade 2006-2015: the silesian stroke registry. Neuroepidemiology. 2018;50(3-4): $183-94$.

7 Sacco RL, Wolf PA, Kannel WB, McNamara PM. Survival and recurrence following stroke. The Framingham study. Stroke. 1982;13(3): $290-5$.
8 Vernino S, Brown RD Jr, Sejvar JJ, Sicks JD, Petty GW, O'Fallon WM. Cause-specific mortality after first cerebral infarction: a population-based study. Stroke. 2003;34(8): 1828-32.

9 Dennis MS, Burn JP, Sandercock PA, Bamford JM, Wade DT, Warlow CP. Long-term survival after first-ever stroke: the oxfordshire community stroke project. Stroke. 1993; 24(6):796-800.

10 Romain G, Mariet AS, Jooste V, Duloquin G, Thomas Q, Durier J, et al. Long-term relative survival after stroke: the Dijon Stroke Registry. Neuroepidemiology. 2019;54(6):498-505.

11 Malmivaara A, Meretoja A, Peltola $\mathrm{M}, \mathrm{Nu}-$ merato D, Heijink R, Engelfriet P, et al. Comparing ischaemic stroke in six European countries. The EuroHOPE register study. Eur J Neurol. 2015;22(2):284-91.

12 Sedova P, Brown RD Jr, Zvolsky M, Kadlecova P, Bryndziar T, Volny O, et al. Validation of stroke diagnosis in the national registry of hospitalized patients in the Czech Republic. J Stroke Cerebrovasc Dis. 2015;24(9):2032-8.

13 Sedova P, Brown RD, Zvolsky M, Belaskova S, Volna $\mathrm{M}$, Baluchova $\mathrm{J}$, et al. Incidence of stroke and ischemic stroke subtypes: a community-based study in Brno, Czech Republic. Cerebrovasc Dis. 2020;50(1):54-61.

14 Bryndziar T, Sedova P, Brown RD Jr, Fiserova E, Zvolsky M, Bednarik J, et al. Trends in one month and one year hemorrhagic stroke case fatality rates in the Czech Republic between 1998 and 2015. J Stroke Cerebrovasc Dis. 2020;29(6):104762.
15 Adams HP, Bendixen BH, Kappelle LJ, Biller J, Love BB, Gordon DL, et al. Classification of subtype of acute ischemic stroke. Definitions for use in a multicenter clinical trial. TOAST. Trial of Org 10172 in acute stroke treatment. Stroke. 1993;24(1):35-41.

16 Aho K, Harmsen P, Hatano S, Marquardsen J, Smirnov VE, Strasser T. Cerebrovascular disease in the community: results of a WHO collaborative study. Bull World Health Organ. 1980;58(1):113-30.

17 Yeramaneni S, Kleindorfer DO, Sucharew H, Alwell K, Moomaw CJ, Flaherty ML, et al. Hyperlipidemia is associated with lower risk of poststroke mortality independent of statin use: a population-based study. Int J Stroke. 2017;12(2):152-60

18 Milionis HJ, Giannopoulos S, Kosmidou M, Panoulas V, Manios E, Kyritsis AP, et al. Statin therapy after first stroke reduces 10 year stroke recurrence and improves survival. Neurology. 2009;72(21):1816-22.

19 Flach C, Elstad M, Muruet W, Wolfe CDA, Rudd AG, Douiri A. The impact of pre- and post-stroke statin use on stroke severity and long-term outcomes: a population-based cohort study. Cerebrovasc Dis. 2019;47(5-6): 260-7.

20 Arboix A, García-Eroles L, Oliveres M, Targa C, Balcells M, Massons J. Pretreatment with statins improves early outcome in patients with first-ever ischaemic stroke: a pleiotropic effect of statins or a beneficial effect of hypercholesterolemia? BMC Neurol. 2010;10:47. 
21 Ong CT, Wong YS, Wu CS, Su YH. Atrial fibrillation is a predictor of in-hospital mortality in ischemic stroke patients. Ther Clin Risk Manag. 2016;12:1057-64.

22 Kongbunkiat K, Kasemsap N, Travanichakul S, Thepsuthammarat K, Tiamkao S, Sawanyawisuth K. Hospital mortality from atrial fibrillation associated with ischemic stroke: a national data report. Int J Neurosci. 2015; 125(12):924-8.

23 Keller K, Geyer M, Münzel T, Ostad MA, Gori T. Impact of atrial fibrillation on in-hospital mortality of ischemic stroke patients and identification of promoting factors of atrial thrombi - results from the German nationwide inpatient sample and a single-center retrospective cohort. Medicine. 2019;98(4): e14086.
24 Kaarisalo MM, Immonen-Räihä P, Marttila RJ, Salomaa V, Kaarsalo E, Salmi K, et al. Atrial fibrillation and stroke. Mortality and causes of death after the first acute ischemic stroke. Stroke. 1997;28(2):311-5.

25 Kaarisalo MM, Immonen-Räihä P, Marttila RJ, Lehtonen A, Salomaa V, Sarti C, et al. Atrial fibrillation in older stroke patients: association with recurrence and mortality after first ischemic stroke. J Am Geriatr Soc. 1997; 45(11):1297-301.

26 Candelise L, Pinardi G, Morabito A. Mortality in acute stroke with atrial fibrillation. The Italian Acute Stroke Study Group. Stroke. 1991;22(2):169-74.
27 Chu CH, Weng WC, Su FC, Peng TI, Chien $\mathrm{YY}, \mathrm{Wu} \mathrm{CL}$, et al. Association between atrial fibrillation and three-year mortality in nondiabetic patients with acute first-ever ischemic stroke. J Stroke Cerebrovasc Dis. 2016; 25(11):2660-7.

28 Joakimsen O, Bønaa KH, Mathiesen EB, Stensland-Bugge E, Arnesen E. Prediction of mortality by ultrasound screening of a general population for carotid stenosis: the Tromsø Study. Stroke. 2000;31(8):1871-6.

29 RES-Q - An ESO Quality Registry. Brno: St. Anne's University Hospital Brno - International Clinical Research Center [Internet]. RES-Q - An ESO Quality Registry; 2021 [cited 2021 Sep 8]. Available from: https://qualityregistry.eu. 